\title{
Using optimisation to suggest alternative supply chains in the context of industrial symbiosis
}

\author{
Florian Stock ${ }^{\text {a b }}$, Simon Dunstall ${ }^{\text {b }}$, Melanie Ayre ${ }^{\text {b }}$, Andreas Ernst ${ }^{\text {b }}$, Asef Nazari ${ }^{\text {b }}$, \\ Dhananjay Thiruvady ${ }^{\mathrm{b}} \mathrm{c}$ and Sarah King ${ }^{\mathrm{d}}$ \\ ${ }^{\mathrm{a}}$ Technical University, Berlin, Germany
${ }^{\mathrm{b}}$ Commonwealth Scientific and Industrial Research Organisation, Clayton, Australia \\ ${ }^{\mathrm{c}}$ Bayesian Intelligence, Melbourne, Australia \\ ${ }^{\mathrm{d}}$ Commonwealth Scientific and Industrial Research Organisation, Geelong, Australia \\ Email: simon.dunstall@csiro.au
}

\begin{abstract}
The concept of firms exchanging materials, energy, water, and/or by-products in a collective approach to competitive advantage is called Industrial Symbiosis (IS). It was first described by Frosch and Gallopoulos in 1989, and in the same year, scientists uncovered an extensively intertwined network of companies from different industries in Kalundborg, Denmark that was a realisation of Frosch and Gallopoulos' ideas. In IS, an unresolved research question relates to whether IS can be designed "ground up" into an industrial system (such as via planned eco-parks), or whether it can only occur "organically" through more serendipitous business interactions.

Given data on a defined set of businesses, quantified amounts of input and raw materials, and material transformation opportunities, our interest is on the use of computation and optimisation techniques in order uncover the potential for IS amongst firms, and to suggest for a single business which potential partners the business should target in order to build a network that could realise this potential. The transformation opportunities are modelled as processes in which a business, consortium, or external agent (such as a local authority) could invest, to enable materials to be transformed from lower to higher value. Examples might include (re-)manufacturing processes, a shared space for materials aggregation, or compressing and baling machinery that could increase waste density and so reduce storage and transportation costs for a material.

To formulate the problem, we consider the potential material flows between businesses and processes, using a linear distance model to describe transportation costs; market values to describe materials that are desirable inputs, and additional sourcing and sinking costs to obtain materials from outside of the network or dispose of surplus materials to landfill. The model is for a single time span, and as such we need to ensure that process investments do not lead to an amplification effect from the total investment cost not being amortized in the considered period.

Results are then presented for each participating business in terms of the origins, destinations and investments that describe the subgraph of material flows that maximises their financial return. For the proposed overall network to be commercially viable, each participant must show a positive return; as such we can demonstrate the difference between the set of local optima and the potential global optimal decisions, thereby uncovering the ideal opportunities for social or government investment.
\end{abstract}

Keywords: Industrial ecology, supply chain optimisation, operations research 


\section{INTRODUCTION}

The cost of disposing industrial waste into landfill has risen considerably in recent times for businesses in Australia. In response, businesses can seek to reduce waste disposal costs by reducing the volume of waste they send to landfill: possibly by finding alternative destinations for waste material. Diverting waste from landfill, or taking non-virgin ex-waste material as packaging or manufacturing process inputs, also can offer environmental advantages and assist a supply chain or industry to lift its resource productivity. Small to Medium-sized Enterprises (SMEs) in manufacturing, logistics and distribution can reduce their landfill waste disposal costs through change and innovation, but in many cases businesses are not aware of their options, lack data that would support a business cases, and do not have the resources needed to seek suitable business partners. A consortium comprising parties from local and state government, as well as CSIRO, has been engaging with SMEs and funding, designing and implementing a web-based system that addresses the SME "knowledge gap" and "partner matchmaking" challenges. This system is referred to as the Advisory System for Processing Innovation and Resource Exchange (ASPIRE). An important part of the ASPIRE system, and the focus of this paper, is network optimisation functionality which is used to form alternative supply chains (businesses, processes, waste streams and material flows) around an SME based on a detailed numerical understanding of materials, manufacturing processes and logistics.

The concept of Industrial Symbiosis (IS) was first described by Frosch and Gallopoulos (1989). IS can be defined as the concept of firms exchanging materials, energy, water, and/or by-products in a collective approach to competitive advantage (Chertow, 2004). The natural drivers for IS are mainly financial, because waste exchanges and input substitutions lead to financial savings, but other possible direct benefits are environmentallyfocused and/or address supply security. Onwards from the time that Frosch and Gallopoulos proposed the IS concept, researchers have uncovered networks of companies engaged in IS in various sites around the world. The deliberate creation of IS networks, such as by way of "eco-industrial parks" (EIPs), has been difficult: e.g., Chertow (2007) reviewed 15 projects in the USA that were commenced under the President's Council on Sustainable Development, and found that after ten years almost all had failed or had reverted to a conventional industrial park concept.

The cases of industrial ecology in Australia can be divided into three categories according to Corder et al. (2014): heavy industrial areas, EIPs and waste exchange networks. In the category of EIPs some projects are reported as successful whereas others failed to prove their viability. Waste exchange networks are typically internet-based and link businesses often for short term waste exchanges. Corder et al. (2014) measured activity on Australian waste exchange websites and determined that almost all had low levels of activity. A successful example of an industrial symbiosis intervention programme is NISP in the United Kingdom (International Synergies Limited, 2014). NISP revolves around raising awareness of potential synergies between businesses by holding workshops and making IS expertise available for finding and implementing exchanges. The national programme was launched in 2005 and by 2009 included a network of 12,500 business members and employed 60 full-time "Industrial Symbiosis Practitioners" (Laybourn and Morrissey, 2009). One of the ingredients of NISP's success has been the assembly of a database of established waste exchanges, which is used to inform businesses of the possibilities and benefits of IS.

\section{Mathematical Models of Industrial Symbiosis}

Different techniques have been proposed for modelling and designing IS and IE systems. Najm et al. (2002) developed a linear programming (LP) model that optimises economical and ecological outcomes at a regional level subject to satisfying material mass balances. The graph-based representation consists of generation nodes, processing facilities, treatment facilities and landfills. Karlsson and Wolf (2008) use mathematical modelling in an attempt to prove that integrated industrial systems are profitable compared to stand alone solutions. The problem is modelled as a network where arcs represent flows and nodes represent a processing unit or line. (Cimren et al., 2011) propose a more general optimisation model that minimises a multiobjective consisting of costs and environmental impact. The model is a network flow approach where binary variables are used to model fixed costs at arcs and nodes. The solution of all preceding LP/MIP models did not pose computational challenges because the network representation consisted of at most 150 nodes, and often less.

A very detailed simulation model that consists of submodels is ORWARE, a model for organic waste handling in urban areas (Dalemo et al., 1997; Eriksson et al., 2002). Cao et al. (2009) present an agent-based model with three types of agents: factory agents seek to produce products thereby maximizing their profit; consumer agents meet their individual demand at lowest cost; and environment agents provide the factory agents with 
raw materials and absorb waste. The model is tested in a case study of a hypothetical eco-industrial park of chemical industries based on an existing EIP in Sichuan, China. Agent-based modelling has also been used as part of an IS-focused intermediation tool (Batten, 2009). Narrowly-focussed models containing deep details of chemical and physical processes have been developed, e.g., for bioethanol (Gonela and Zhang, 2014).

Based on the published literature on IS practice we propose that systematically establishing IS either requires the intensive engagement of businesses and the orchestration of connections in the style of NISP, or if done via lower-cost methods and with less direct intervention, a digitally-oriented solution which emphasizes the provision of technical information and the suggestion of potential partners for long-term business relationships that are relevant to an industrial enterprise. We are pursuing the latter approach, to embed in a web-based system, and within this an important capability is a means of devising technically-plausible alternatives for material flows, processes and supply networks which have the potential to be financially viable.

\section{SUPPLY NETWORK OPTIMISATION IN THE CONTEXT OF INDUSTRIAL SyMbiOSIS}

We seek an optimisation-based approach to generating IS-oriented supply networks. We do this for a use-case where a particular small-medium enterprise (a Subject SME) is seeking alternative processing and business partnership suggestions from the decision-support system. For the Subject SME the amounts and ratios of input and output materials are known. The materials we refer to as commodities and the global set of commodities covers all materials that are input or output of at least one process known to the decision-support system. Quality, shape, colour and other attributes can be very important when distinguishing between suitable and unsuitable materials for (re-)manufacturing processes and recycling. An ontology for commodities has therefore been devised which captures the necessary attributes. This allows us to represent, for example, a case of a process that transforms cooking oil into fuel, and can use sunflower oil or olive oil as an input, but not machine oil. Some commodities can be distinguished as end products that must not be further considered for further processing in the network: e.g., refined and marketable raw materials or to elaborate consumer goods.

We define a process as a mapping of input to output commodities according to a known ratio. We distinguish between transformation processes which transform materials and have different input and output commodities and/or non-unit ratios, and abstract processes which do not transform materials. A transformation process represents machinery, reactors and other technology for processing and value-adding. Abstract processes represent activities such as landfill, waste management services and markets external to an SME supply network. Abstract process outputs are treated as peripheral to the supply network around a Subject SME.

If an alternative supply network for the Subject SME makes use of a transformation process that either is not available amongst businesses open to IS arrangements in the region, or is present but of insufficient capacity for the Subject SME, then we can inject an instance of the process into the network, and represent the investment as a fixed component of cost in the objective function. In the optimisation, a process can be injected in a supply network if its outputs are an input to a process of a known business, it is an abstract process, or there is some chain of value-adding transformation processes that lead to a process of a known business.

A transformation process can occur in the real world with different throughput capacities and different fixed and variable costs. We define for each process one or more implementation options with minimum and maximum throughput capacities. For transformation processes this essentially models processing systems and equipment of different sizes. Abstract processes have variable costs only, i.e., there is no fixed cost charged for the existence of the process in the network. Implementation options for abstract processes relate to different volume-related material handling cost structures for the network of the Subject SME, i,e., economies of scale.

Capacities in the supply network need to be compatible. This drives the selection of implementation options, and can be used to represent bounded external markets when associated with abstract processes. For example, consider that the Subject SME emits 100t of timber waste per period. Based on data on existing businesses, the optimisation might identify possible input substitutions for other known SMEs for 20t of this timber per period. This generates a cost reduction or nett revenue gain for the Subject SME, and no additional capital cost for the network. A known transformation process of turning wood waste into fuel may have an implementation option for 30t of waste timber input per period, and this can be injected into the network, bringing with it fixed and variable cost components for the network. An abstract process might embody further knowledge that 40t of timber per period (as the maximum-sized implementation option) could be sold outside of the region for some productive purpose. The remaining $10 \mathrm{t}$ per period will then have to flow to an abstract process representing landfill, balancing the flows and contributing a corresponding cost for the supply network of the Subject SME.

A supply network for the Subject SME is represented as a graph, with flows of commodities on arcs, and nodes 
representing process implementation options as well as sources and sinks for each commodity. Arcs connect processes with inputs and outputs of the same commodity, and flows on these arcs represent a magnitude of commodity flow per unit time. It can be shown that the NP-hard longest path problem is a special case of our supply network design problem — thus our problem is provably NP-hard.

\section{Optimisation using an Mixed Integer Program}

In our first algorithm, the objective is to minimise cost for the subject SME by resolving flows on a graph $\mathcal{G}$ of all potential processes and material flows in a supply network around the subject SME. This optimal solution yields a supply network design. For each arc $a$ and commodity $k$ we introduce a continuous flow variable $x_{a, k}$ which defines the amount of each commodity that flows between the source and destination nodes (i.e., process instances) per unit time (i.e., per period). Commodity flow through nodes representing transformation processes is not balanced: rather, flows are governed by fixed ratios between input and output commodities. We introduce continuous execution variables $z_{p, o}$, where $(p, o)$ identifies the node in which $p$ is the process and $o$ is the implementation option. Equations 1 and 2 are flow conservation and commodity transformation constraints.

$$
\begin{aligned}
\sum_{a \in \operatorname{in}(p)} x_{a, k} & =\sum_{o \in \mathcal{O}_{p}} z_{p, o} \alpha_{p, k} \quad \forall k \in \mathcal{C}, p \in \mathcal{P} \cup \mathcal{T} \\
\sum_{a \in \text { out }(p)} x_{a, k} & =\sum_{o \in \mathcal{O}_{p}} z_{p, o} \beta_{p, k} \quad \forall k \in \mathcal{C}, p \in \mathcal{P} \cup \mathcal{S}
\end{aligned}
$$

In this, $\mathcal{C}$ is the set of commodities, $\mathcal{P}$ is the set of nodes representing processes, $\mathcal{S}$ and $\mathcal{T}$ are commodity sources and sinks, $\mathcal{O}_{p}$ are implementation options for process $p \in \mathcal{P}$, and $\alpha_{p, k}$ and $\beta_{p, k}$ are the relative ratios of flow for commodity $k$ for a unit execution of process $p$ (i.e., for $z_{p, o}=1$ ). For conversion processes, no particular ratios apply and a simple flow conservation constraint is used (i.e., mass in equals mass out).

Each implementation option has a minimum $c_{p, o}^{\min }$ and a maximum $c_{p, o}^{\max }$ process execution capacity (zero and unbounded, respectively, if not specified), and executed flow must be within the throughput limits:

$$
c_{p, o}^{\min } \delta_{p, o} \leq z_{p, o} \leq c_{p, o}^{\max } \delta_{p, o} \quad \forall p \in \mathcal{P}, o \in \mathcal{O}_{p}
$$

where a binary variable $\delta_{p, o}$ indicates if a process option $o$ is used for process $p$ (i.e., $z_{p, o}>0$ ). This infers that an option can only be used once per process, but multiple options can be used for the same process, i.e., at most one "machine of each size" can be bought. In an alternative formulation we could allow $\delta_{p, o}$ to be a general integer variable, but for most practical cases we do not expect this to be necessary.

We want to minimise cost from the perspective of the Subject SME which has a process $P_{0}$. We approximate this as the sum of costs for input materials, costs for by-products (which could be a revenue, so negativevalued) and costs for implementing transformation processes that are not yet present in the supply network. Each process option has a fixed cost and a variable cost component. At sources and sinks the fixed cost component is 0 and variable costs are the market price of the commodity. We write $\Phi_{s_{k}, o}$ for the market buying price of commodity $k$ at option $o$ and $\Phi_{t_{k}, o}$ for the market selling price of commodity $k$ at option $o$. At transformation process instances $(p, o)$ the cost is split further into: $E Q_{p, o}$ as the (levelized) equipment cost; $L C_{p, o}$ (levelized) labour and commissioning cost; $O M_{p, o}$ as the per-period operations and maintenance cost; and $U P C_{p, o}$ unit execution cost. Given this, the objective function is:

$$
\begin{aligned}
\text { minimise } & \sum_{p \in \mathcal{P}} \sum_{o \in \mathcal{O}_{p}} \delta_{p, o}\left(E Q_{p, o}+L C_{p, o}+O M_{p, o}\right) \\
+ & \sum_{p \in \mathcal{P}} \sum_{o \in \mathcal{O}_{p}} z_{p, o} \cdot U P C_{p, o}+\sum_{k \in \mathcal{C}} \sum_{o \in O_{s_{k}}} z_{s_{k}, o} \cdot \Phi_{s_{k}, o}+\sum_{k \in \mathcal{C}} \sum_{o \in O_{t_{k}}} z_{t_{k}, o} \cdot \Phi_{t_{k}, o}
\end{aligned}
$$

Demand for the production at the Subject SME must be satisfied, so we introduce an implementation option $\hat{o}$ for $P_{0}$ that reflects via $\alpha_{P_{O}, \hat{o}}^{\{\min , \max \}}$ the required volumes of inputs and outputs, and we set $z_{P_{0}, \hat{o}}=1$.

The capability to buy any commodity from its sources in $G$ allows for an unwanted case where flows from a source to a sink that are completely unrelated to $P_{0}$ can be profitable and therefore part of an optimal solution. These flows are irrelevant and undesirable, but unless they cause major numerical scaling issues, can be permitted and then simply ignored when translating the MIP solution into a set of supply network 
suggestions. More importantly, we permit bringing "new mass" into the network from external sources because transformation processes can need inputs that cannot be sourced from the pre-existing network of the Subject SME. However, this mass should be constrained to be within some maximum proportion of the mass originally associated with $P_{0}$, otherwise we can generate unreasonable solutions (e.g., a goods manufacturer builds a metal recycling plant in order to recycle a small amount of steel offcuts). We reformulate our model such that the maximum ratio of mass that originates from the subject SME to the mass that is brought into the system is constrained by a parameter $\rho \in[0,1]$. For this purpose, we duplicate $G$ to form a graph $H$, and then make the commodity sources and sinks common to each. All flows in $G$ conserve the mass that is processed by $P_{0}$, whereas all flows in $H$ are for mass that is sourced and sunk upstream or downstream of $P_{0}$. The original flow variables "split" into flow variables for $G$ and $H$, ditto for process execution variables. The $\delta$ variables are common because the choice of implementation option is common for the two graphs. Flows originating from sources must be in the $H$ graph, except for are commodities that are input to $P_{0}$. For all other transformation processes we restrict the maximum ratio of mass flow in $G$ and in $H$ with the parameter $\rho \in[0,1]$. If $\rho$ is close to zero, almost all mass in the supply network must pass through $P_{0}$. If $\rho$ is close to one, much additional mass can enter and leave the supply network. Further details of this reformulation are straightforward to derive.

When there are many commodities and processes of relevance, graph $G$ (or, $G$ and $H$ ) can be complex. To simplify $G$ we can introduce a warehouse process for each commodity, and instead of connecting all pairs of processes with matching inputs and outputs, we connect each to the warehouses for their commodities. This reduces the number of arcs significantly, while introducing only a few additional nodes. The disadvantage in this approach is that point-to-point material transportation costs and capacities cannot be captured.

\section{OPTIMISATION USING A BINARY DECISION DiAgRAM}

In our second algorithm, we use a branch-and-bound approach based on Binary Decision Diagrams (BDDs), e.g., Akers (1978); Becker et al. (2005). For optimisation problems with binary decisions, BDDs have nodes representing states consisting of choices and possible future decisions, and weights can be given to the arcs to represent an objective function. A longest path in the decision diagram represents a feasible solution. For simpler problems, the weight of the path is the objective function value. The discrete decisions in our problem are which transformation processes to include, and which implementation options to assign to them. At a BDD node, solving the remaining LP on the restricted network yields a solution. We build on the idea of BDDs to generate a custom branch-and-bound algorithm.

We define a state $s$ as a set of processes with their implementation options. $\mathcal{P}_{s}$ is the set of processes involved in state $s$ and $\mathcal{C}_{p}$ is the set of commodities that are input or output of a process $p$. A state implies a subgraph in $G$, via the node set $\bigcup_{p \in \mathcal{P}_{s}} \mathcal{C}_{p} \cup \mathcal{P}_{s}$. Solving the LP in the subgraph generates a restricted feasible solution and therefore an upper bound on the objective function (4). A lower bound can be obtained by solving an LP on the complete network $G$, with relevant $\delta_{p, o}$ variables set to $\{0,1\}$ according to $s$, and relaxed otherwise.

Each node in the BDD represents a state, and the binary choices are to include or exclude an option or a whole process. For the purposes of the algorithm we devise a complete ordering of processes with implementation options. Since we expect solutions to contain short supply chains, we choose an ordering of the processes as indicated by proximity to the Subject SME in $G$. The implementation options for a process can be ordered according to their values in an initial LP relaxation. Let $\sigma=\sigma_{1}, \ldots, \sigma_{n}$ be an ordering of processes and implementation options. $\sigma_{i} \in \mathcal{P} \cup \mathcal{O}, n=|\mathcal{P}| \cdot|\mathcal{O}|+|\mathcal{P}|$. Stepping through the BDD means making a decision for each $\sigma_{i}$ in the provided order. The decision to eliminate a process can make other processes unreachable. Decisions to include or eliminate an option have an effect on other options and processes via capacities: if the maximum capacity that can reach a node is lower than the minimum capacity of an option, that option cannot be implemented (e.g, refer Figure 1). For a shorter notation, we use the following convention: branching from $\sigma_{i}$ to $\sigma_{j}$ adds option $\sigma_{j}$ to the current set of processes with options, and sets $\sigma_{k}=0$ for all $i \leq k \leq j$. This means that skipping options in the devised ordering implies rejecting these options. After each choice the set of choices at a node is updated with respect to the rules mentioned above. Different choices can lead to the same state, however, we cannot put weights on the arcs to compute a solution value. For upper bounds we can round all options that have not been set yet, and then solve the LP to compute the flows.

The tree can grow exponentially, though we expect it to be wide and flat (this allowing massive parallelisation). Considering that two LPs need to be solved at each node of the BDD to get upper and lower bounds, exploring the complete tree can be computationally very expensive. We have explored the use of beam search, integer rounding and Ant Colony Optimisation (ACO) for setting $\delta_{p, o}$ variables in LP relaxations when seeking upper bounds. 
F. Stock et al, Using optimisation to suggest alternative supply chains in the context of industrial symbiosis
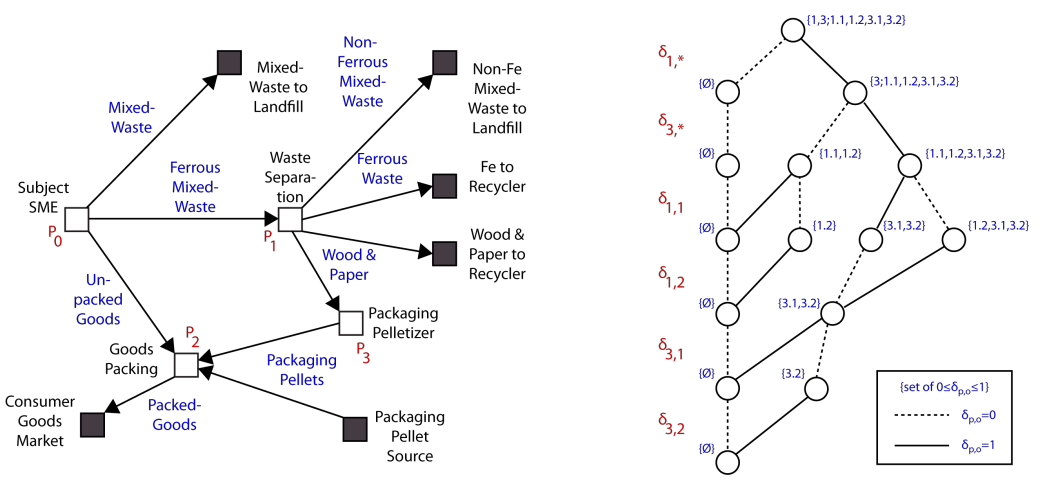

Figure 1. An example process graph (left) and corresponding Binary Decision Diagram (right)

\section{Computational Comparison of Algorithms}

In exploring the computational difficulty of our supply network optimisation problem we use synthesized data because sufficient real-world data has not yet been assembled. We constructed ten scenarios with randomlygenerated processes, implementation options and SMEs. For implementation options, with increasing throughput capacities the equipment cost increases and the unit production cost decreases. The scenarios vary in the number of processes, commodities, options, and inputs and outputs. Table 1 lists the scenarios with their properties, as well as the size of the resulting MIPs. The graph representation of the problem has a low density of less than $1 \%$ for all instances. For each scenario group we create two instances by selecting a subject SME and setting its demand. We solve the instances with CPLEX (for the MIP-based approach) and with the heuristics implemented in Python 2.7 (for the BDD approach). For each scenario there is a time limit: 600, 1200, 1800, 3600 and 3600 seconds for scenarios A to $\mathbf{E}$ respectively. These limits are far too generous for the ASPIRE use case, because users might not be prepared to wait for long periods, but parallelisation could speed up all of the methods. Parallel computation is difficult in Python and this had a strong influence on the way we designed the details of the meta-heuristics (i.e., within the given time limits only a small number of LPs could be solved).

Table 1. Scenario parameters and MIP size

\begin{tabular}{|c|c|c|c|c|c|c|c|}
\hline Scenario & \#Processes & \#Commodities & $\begin{array}{c}\text { Mean \#(inputs and } \\
\text { outputs) per process }\end{array}$ & $\begin{array}{c}\text { Mean \#options per } \\
\text { process }\end{array}$ & MIP \#variables & MIP \#binaries & MIP \#constraints \\
\hline A1 & 1000 & 2000 & 3 & 4 & 33,187 & 8,312 & 28,248 \\
\hline A2 & 1000 & 2000 & 4 & 9 & 41,592 & 12,447 & 32,430 \\
\hline A3 & 1000 & 2000 & 8.5 & 4 & 43,903 & 8,466 & 33,975 \\
\hline A4 & 1000 & 1000 & 3.5 & 5 & 25,096 & 6,551 & 19,059 \\
\hline A5 & 1000 & 500 & 3 & 5 & 20,396 & 5,500 & 13,975 \\
\hline B1 & 2500 & 4000 & 3.5 & 4 & 75,432 & 19,090 & 61,961 \\
\hline B2 & 2500 & 2500 & 3.5 & 4 & 62,361 & 16,194 & 47 \\
\hline C & 3500 & 3500 & 3 & 5 & 87,590 & 22,859 & 63 \\
\hline D & 4000 & 4000 & 4 & 5 & 100,236 & 26,085 & 76,548 \\
\hline E & 5000 & 5000 & 3.5 & 4 & 124,826 & 36,101 \\
\hline
\end{tabular}

Primal rounding, beam search and ACO do not provide lower bounds but they do return feasible upper-bound solutions. Table 2 shows the run-time duration for which solutions obtained from BDD heuristics are better than from CPLEX. The fastest way to obtain a good feasible solution is to use the BDD with primal rounding, and the heuristic solutions are always better at the start of a run. In most scenarios this holds for more than 100 seconds. For scenarios $\mathbf{B}$ to $\mathbf{E}$ all heuristic solutions are better than CPLEX at the end of the run.

\section{CONCLUSIONS ANd ONgOING RESEARCH}

The model development and computational experiments have demonstrated that network optimisation techniques can be applied to the task of generating insight into the possibilities for Industrial Symbiosis. The computational work that has been carried out to date has exclusively considered single-threaded algorithms, even though the BDD-based approach offers very significant opportunities for parallelisation. The BDD-based algorithms have distinct advantages over the MIP-based approach. Not only do they outperform the MIP in our tests, but they offer good potential for parallelisation, feasible intermediate solutions could be displayed to users, and the algorithms terminated at a time limit with a feasible solution. We plan to investigate the parallel processing opportunities, and when a sufficient body of data from real-world industrial operations has been assembled, to assess algorithm performance on this real-world data. Further research should also consider pos- 
F. Stock et al, Using optimisation to suggest alternative supply chains in the context of industrial symbiosis

Table 2. The duration in seconds for which the BDD heuristics provided a better solution than the MIP

\begin{tabular}{|c|c|c|c|}
\hline Scenario & Primal Rounding & Beam Search & ACO \\
\hline A1 (Instance 1) & 182.74 & 318.48 & 318.48 \\
\hline A1 (Instance 1) & 371 & 397.1 & 371 \\
\hline A2 (Instance 1) & 324.57 & 324.57 & 324.57 \\
\hline A2 (Instance 2) & 420.31 & 420.31 & 420.31 \\
\hline A3 (Instance 1) & 563.43 & 563.43 & 563.43 \\
\hline A3 (Instance 2) & 606 & 606 & 606 \\
\hline A4 (Instance 1) & 303.25 & 570.99 & 303.25 \\
\hline A4 (Instance 2) & 599.4 & 599.4 & 599.4 \\
\hline A5 (Instance 1) & 321.86 & 586.05 & 321.86 \\
\hline A5 (Instance 2) & 41.82 & 550 & 41.82 \\
\hline
\end{tabular}

sibilities for direct user interaction and feedback into the process of generating alternative supply networks, as well as the integration into the BDD heuristic of some relevant real-world subproblems associated with transport or other operational details that could be evaluated at each decision tree node.

\section{REFERENCES}

Akers, S. (1978, June). Binary decision diagrams. IEEE Transactions on Computers C-27(6), 509-516.

Batten, D. F. (2009, April). Fostering industrial symbiosis with agent-based simulation and participatory modeling. Journal of Industrial Ecology 13(2), 197-213.

Becker, B., M. Behle, F. Eisenbrand, and R. Wimmer (2005). Bdds in a branch and cut framework. In Experimental and Efficient Algorithms, pp. 452-463. Springer.

Cao, K., X. Feng, and H. Wan (2009, September). Applying agent-based modeling to the evolution of ecoindustrial systems. Ecological Economics 68(11), 2868-2876.

Chertow, M. R. (2004). Industrial symbiosis. Encyclopedia of energy 3, 407-415.

Chertow, M. R. (2007). Uncovering industrial symbiosis. Journal of Industrial Ecology 11(1), 1130.

Cimren, E., J. Fiksel, M. E. Posner, and K. Sikdar (2011). Material flow optimization in by-product synergy networks. Journal of Industrial Ecology 15(2), 315332.

Corder, G. D., A. Golev, J. Fyfe, and S. King (2014, March). The status of industrial ecology in australia: Barriers and enablers. Resources 3(2), 340-361.

Dalemo, M., U. Sonesson, A. Bjrklund, K. Mingarini, B. Frostell, H. Jnsson, T. Nybrant, J.-O. Sundqvist, and L. Thyselius (1997, September). ORWARE a simulation model for organic waste handling systems. part 1: Model description. Resources, Conservation and Recycling 21(1), 17-37.

Eriksson, O., B. Frostell, A. Bjrklund, G. Assefa, J. O. Sundqvist, J. Granath, M. Carlsson, A. Baky, and L. Thyselius (2002, November). ORWAREa simulation tool for waste management. Resources, Conservation and Recycling 36(4), 287-307.

Frosch, R. A. and N. E. Gallopoulos (1989). Strategies for manufacturing. Scientific American 261(3), 144152.

Gonela, V. and J. Zhang (2014, February). Design of the optimal industrial symbiosis system to improve bioethanol production. Journal of Cleaner Production 64, 513-534.

International Synergies Limited (2014, July). National industrial symbiosis programme. http://www . nispnetwork. com/.

Karlsson, M. and A. Wolf (2008, September). Using an optimization model to evaluate the economic benefits of industrial symbiosis in the forest industry. Journal of Cleaner Production 16(14), 1536-1544.

Laybourn, P. and M. Morrissey (2009). National industrial symbiosis programme the pathway to a low carbon sustainable economy. Technical Report ISBN: 97809564235 1 1, International Synergies Ltd.

Najm, M. A., M. El-Fadel, G. Ayoub, M. El-Taha, and F. Al-Awar (2002, February). An optimisation model for regional integrated solid waste management II. model application and sensitivity analyses. Waste Management \& Research 20(1), 46-54. PMID: 12020095. 